\author{
M.T. Jenaliyev ${ }^{1}$, M.I. Ramazanov ${ }^{2, *}$, A.O. Tanin ${ }^{2}$ \\ ${ }^{1}$ Institute of Mathematics and Mathematical Modeling, Almaty, Kazakhstan; \\ ${ }^{2}$ Buketov Karaganda University, Karaganda, Kazakhstan \\ (E-mail: muvasharkhan@gmail.com,ramamur@mail.ru,tanin.alibek@gmail.com)
}

\title{
To the solution of the Solonnikov-Fasano problem with boundary moving on arbitrary law $x=\gamma(t)$.
}

\begin{abstract}
In this paper we study the solvability of the boundary value problem for the heat equation in a domain that degenerates into a point at the initial moment of time. In this case, the boundary changing with time moves according to an arbitrary law $x=\gamma(t)$. Using the generalized heat potentials, the problem under study is reduced to a pseudo-Volterra integral equation such that the norm of the integral operator is equal to one and it is shown that the corresponding homogeneous integral equation has a nonzero solution.

Key words: heat equation, moving boundary, degenerating domain, pseudo-Volterra integral equation.
\end{abstract}

\section{Introduction}

In many practically important engineering problems, the process of forming a temperature field in the structure under study is accompanied by the removal of a part of the substance from the surface, which leads to a change in its boundaries over time. The need to take into account the of the boundaries mobility of the studied domain significantly complicates the solution of the corresponding problems [1-5].

For example, in mathematical modeling of thermophysical processes in an electric arc of high-current disconnecting devices, the heat equation is used, which takes into account the effect of heat sources in the arc and the effect of contracting the axial section of the arc in the cathode region into the contact spot [6]. Moreover, the diameter of the contact spot is much less than the section diameter of the developed column of the arc, which makes it possible to consider it as a mathematical point. At the initial moment of time, the contacts are in a closed state and solution domain of the problem is absent; then, the solution domain changes over time according to the conditions for opening the contacts.

From a mathematical point of view, the singularity of the problem under consideration lies, firstly, in the presence of a moving boundary, and secondly, in the degeneration of the solution domain at the initial moment $[7,8]$. Problems in domains with moving boundaries are also relevant in modeling physical processes in a gas discharge plasma, during melting of electrical contacts, the effect of an electric arc on contacts, in studying the problems of thermal shock in domains with a moving boundary, in solving a number of problems in hydromechanics [9-13].

Applying the method of generalized heat potentials, a number of similar problems can be reduced to the solution of sinqular Volterra type integral equations of the second kind. It is essential here that if in the boundary value problem the variable domain does not degenerate into a point at the initial moment of time, then the integral equation equivalent to it is solved by the method of successive approximations. If the domain degenerates into a point at the initial moment of time, then the integral equation of the boundary value problem has a singularity, which is that the integral from the kernel tends to unity as the upper limit of integration tends to the lower one, and this means that the method of successive approximations is not applicable to it.

\section{Statement of the problem}

Let's study the solvability issues of the following boundary value problem:

$$
\frac{\partial u}{\partial t}-a^{2} \frac{\partial^{2} u}{\partial x^{2}}=f(x, t), \quad\{0<x<\gamma(t), t>0\}
$$

\footnotetext{
${ }^{*}$ Corresponding author.
}

E-mail:ramamur@mail.ru 


$$
\left.\frac{\partial u}{\partial x}\right|_{x=0}=u_{0}(t), \quad \frac{d \tilde{u}(t)}{d t}+\left.\frac{\partial u}{\partial x}\right|_{x=\gamma(t)}=u_{1}(t)
$$

where $\tilde{u}(t)=u(\gamma(t), t), \quad \gamma(0)=0$ for $\gamma(t)=\left[t\left(1+\alpha_{0}(t)\right)\right]^{\omega}, \quad \omega>\frac{1}{2}$.

Function $\gamma(t):(0, \infty) \rightarrow(0, \infty)$ satisfies the following conditions:

1. asymptotics of the function $\gamma(t)$ as $t \rightarrow 0$ and as $t \rightarrow \infty$ has the form $t^{\omega}$, where $\omega>\frac{1}{2}$;

2. starting from some moment of time $t_{1}^{*}$ until moment of time $t_{2}^{*}$ the function $\gamma(t)$ is arbitrary, strictly monotone and one-to-one, i.e. there is a reverse transformation $\gamma^{-1}(t)$.

We introduce the classes of solutions and data of the problem as follows:

$$
\begin{gathered}
\left(x+[\gamma(t)]^{\frac{3}{2 \omega}-1}\right)^{-1} u(x, t) \in L_{\infty}(G), \text { i.e. } \quad u(x, t) \in L_{\infty}\left(G ;\left(x+[\gamma(t)]^{3 / 2 \omega-1}\right)^{-1}\right), \\
f(x, t) \in W_{\infty}^{1,0}\left(G ;[\gamma(t)]^{3 / 2 \omega-1} \exp \left\{[\gamma(t)]^{\frac{2 \omega-1}{\omega}} /\left(4 a^{2}\right)\right\}\right) ; \\
u_{0}(t) \in L_{\infty}\left(R_{+} ;[\gamma(t)]^{-(3 / 2 \omega-1)}\right) ; \quad u_{1}(t) \in L_{\infty}\left(R_{+} ;[\gamma(t)]^{3 / 2 \omega-1}\right) .
\end{gathered}
$$

This kind of boundary value problem (1) arises, for example, in studies of the Stefan problem [14].

\section{Transformation of problem (1) and reduction of it to the integral equation}

Introducing a new unknown function $v(x, t)=\frac{\partial u}{\partial x}$, we transform problem (1)-(2) to the next problem:

$$
\begin{gathered}
\frac{\partial v}{\partial t}-a^{2} \frac{\partial^{2} v}{\partial x^{2}}=\tilde{f}(x, t), \quad\{0<x<t, \quad t>0\} \\
\left.v(x, t)\right|_{x=0}=v_{0}(t),\left.\quad\left(\frac{\partial v}{\partial x}+\frac{1+\gamma^{\prime}(t)}{a^{2}} v\right)\right|_{x=\gamma(t)}=v_{1}(t),
\end{gathered}
$$

where $\tilde{f}(x, t) \equiv \frac{\partial f(x, t)}{\partial x}, \quad v_{0}(t) \equiv u_{0}(t), \quad v_{1}(t) \equiv \frac{u_{1}(t)}{a^{2}}+\left.\frac{f(x, t)}{a^{2}}\right|_{x=\gamma(t)}$.

Remark 1. Each solution to boundary value problem (3)-(4) defines a unique solution (up to a constant) of boundary value problem (1)-(2).

We will find the solution of problem (3)-(4) as the sum of heat potentials [15]:

$$
\begin{aligned}
& v(x, t)=\frac{1}{2 a \sqrt{\pi}} \int_{0}^{t} \int_{0}^{\infty} \frac{1}{(t-\tau)^{1 / 2}} \exp \left\{-\frac{(x-\xi)^{2}}{4 a^{2}(t-\tau)}\right\} \tilde{f}(\xi, \tau) d \xi d \tau+ \\
& +\frac{1}{4 a^{3} \sqrt{\pi}} \int_{0}^{t} \frac{x}{(t-\tau)^{3 / 2}} \exp \left\{-\frac{x^{2}}{4 a^{2}(t-\tau)}\right\} \nu(\tau) d \tau+ \\
& +\frac{1}{2 a \sqrt{\pi}} \int_{0}^{t} \frac{1}{(t-\tau)^{1 / 2}} \exp \left\{-\frac{(x-\gamma(\tau))^{2}}{4 a^{2}(t-\tau)}\right\} \varphi(\tau) d \tau .
\end{aligned}
$$

The function defined by equality (5) satisfies the equation (3) for any functions $\nu(t)$ and $\varphi(t)$, which are still unknown and are to be determined further.

Satisfying solution (5) with boundary conditions (4), we obtain the following integral equation:

$$
\varphi(t)+\int_{0}^{t} K_{\gamma}(t, \tau) \varphi(\tau) d \tau=F(t)
$$

kernel $K_{\gamma}(t, \tau)$ of which can be represented as a sum:

$$
K_{\gamma}(t, \tau)=\sum_{i=1}^{4} K_{\gamma}^{(i)}(t, \tau),
$$


where:

$$
\begin{aligned}
& K_{\gamma}^{(1)}=\frac{1}{2 a \sqrt{\pi}} \frac{\gamma(t)+\gamma(\tau)}{(t-\tau)^{\frac{3}{2}}} \exp \left\{-\frac{(\gamma(t)+\gamma(\tau))^{2}}{4 a^{2}(t-\tau)}\right\} ; \\
& K_{\gamma}^{(2)}=-\frac{1}{2 a \sqrt{\pi}} \frac{\gamma(t)-\gamma(\tau)}{(t-\tau)^{\frac{3}{2}}} \exp \left\{-\frac{(\gamma(t)-\gamma(\tau))^{2}}{4 a^{2}(t-\tau)}\right\} ; \\
& K_{\gamma}^{(3)}=-\frac{1}{a \sqrt{\pi}} \frac{1+\gamma(t)^{\prime}}{(t-\tau)^{\frac{1}{2}}} \exp \left\{-\frac{(\gamma(t)+\gamma(\tau))^{2}}{4 a^{2}(t-\tau)}\right\} ; \\
& K_{\gamma}^{(3)}=\frac{1}{a \sqrt{\pi}} \frac{1+\gamma(t)^{\prime}}{(t-\tau)^{\frac{1}{2}}} \exp \left\{-\frac{(\gamma(t)-\gamma(\tau))^{2}}{4 a^{2}(t-\tau)}\right\} .
\end{aligned}
$$

The free term of equation (6) has the following form:

$$
\begin{gathered}
F(t)=-\frac{a}{\sqrt{\pi}} \int_{0}^{t}\left[\frac{1}{(t-\tau)^{3 / 2}}-\frac{\gamma^{2}(t)}{2 a^{2}(t-\tau)^{5 / 2}}\right] \exp \left\{-\frac{\gamma^{2}(t)}{4 a^{2}(t-\tau)}\right\} v_{0}(\tau) d \tau+ \\
-\frac{\left.1+\gamma^{\prime}(\mathrm{t})\right)}{a \sqrt{\pi}} \int_{0}^{t} \frac{\gamma(t)}{(t-\tau)^{3 / 2}} \exp \left\{-\frac{\gamma^{2}(t)}{4 a^{2}(t-\tau)}\right\} v_{0}(\tau) d \tau+2 a^{2} \cdot v_{1}(t)- \\
-\frac{1}{2 a \sqrt{\pi}} \int_{0}^{t} \int_{0}^{\infty}\left[\frac{\gamma(t)+\xi}{(t-\tau)^{3 / 2}} \exp \left\{-\frac{(\gamma(t)+\xi)^{2}}{4 a^{2}(t-\tau)}\right\}-\frac{\gamma(t)-\xi}{(t-\tau)^{3 / 2}} \exp \left\{-\frac{(\gamma(t)-\xi)^{2}}{4 a^{2}(t-\tau)}\right\}\right] \tilde{f}(\xi, \tau) d \xi d \tau- \\
-\frac{1}{a \sqrt{\pi}} \int_{0}^{t} \int_{0}^{\infty} \frac{1+\gamma^{\prime}(t)}{(t-\tau)^{1 / 2}} \cdot \exp \left\{-\frac{(\gamma(t)-\xi)^{2}}{4 a^{2}(t-\tau)}\right\} \cdot \tilde{f}(\xi, \tau) d \xi d \tau .
\end{gathered}
$$

We will find the solution of integral equation (6) in the class of functions:

$$
[\gamma(t)]^{\frac{3}{2 \omega}-1} \varphi(t) \in L_{\infty}(0, \infty) \text {, т.e. } \varphi(t) \in L_{\infty}\left(0, \infty ;[\gamma(t)]^{\frac{3}{2 \omega}-1}\right) .
$$

For convenience, we represent equation (6) as follows:

$$
\varphi_{1}(t)+\int_{0}^{t}\left[\frac{\gamma(t)}{\gamma(\tau)}\right]^{\frac{3}{2 \omega}-1} K_{\gamma}(t, \tau) \varphi_{1}(\tau) d \tau=F_{1}(t)
$$

where

$$
\varphi_{1}(t)=t^{\frac{3}{2 \omega}-1} \cdot \varphi(t), \quad F_{1}(t)=t^{\frac{3}{2 \omega}-1} \cdot F(t)
$$

Remark 2. ([16], p.183) If the (particular) solution of the integral equation

$$
y(x)+\int_{a}^{x} K(x, t) y(t) d t=f(x)
$$

is given by formula

$$
y(x)=f(x)+\int_{a}^{x} R(x, t) f(t) d t,
$$

then the (particular) solution of the integral equation (with a modified kernel)

$$
y(x)+\int_{a}^{x} K(x, t) \frac{g(x)}{g(t)} y(t) d t=f(x)
$$

is given by the formula

$$
y(x)=f(x)+\int_{a}^{x} R(x, t) \frac{g(x)}{g(t)} f(t) d t .
$$

The same is true for the solutions of the corresponding homogeneous equations.

Such kind of the Volterra integral equations were considered in the papers $[17,18]$.

Note that a feature of integral equation (7) is the following property of the kernel $K_{\gamma}(t, \tau)$ :

$$
\lim _{t \rightarrow 0+} \int_{0}^{t} K_{\gamma}(t, \tau) d \tau=1 .
$$

In order to solve integral equation (7), consider the corresponding characteristic integral equation. 
Characteristic integral equation. Estimates for kernels of integral operators

For integral equation (7) we will construct a characteristic equation

$$
\varphi(t)+\int_{0}^{t}\left[\frac{\gamma(t)}{\gamma(\tau)}\right]^{\frac{3}{2 \omega}-1} K_{h}(t, \tau) \varphi(\tau) d \tau=g(t),
$$

where

$$
\begin{aligned}
& K_{h}(t, \tau)=\sum_{i=1}^{4} K_{h}^{(i)}(t, \tau) \\
& K_{h}^{(1)}(t, \tau)=\frac{1}{2 a \sqrt{\pi}} \cdot \frac{(2 \omega-1)^{\frac{3}{2}}\left([\gamma(\tau)]^{\frac{2 \omega-1}{\omega}} \cdot[\gamma(t)]^{\frac{2 \omega-2}{\omega}}+[\gamma(t)]^{\frac{4 \omega-3}{\omega}}\right)\left\{[\gamma(\tau)]^{\frac{1}{\omega}}\right\}^{\prime}}{\left([\gamma(t)]^{\frac{2 \omega-1}{\omega}}-[\gamma(\tau)]^{\frac{2 \omega-1}{\omega}}\right)^{\frac{3}{2}}} . \\
& \cdot \exp \left\{-\frac{(2 \omega-1)\left([\gamma(t)]^{\frac{2 \omega-1}{\omega}}+[\gamma(\tau)]^{\frac{2 \omega-1}{\omega}}\right)^{2}}{4 a^{2}\left([\gamma(t)]^{\frac{2 \omega-1}{\omega}}-[\gamma(\tau)]^{\frac{2 \omega-1}{\omega}}\right)}\right\} \\
& K_{h}^{(2)}(t, \tau)=-\frac{1}{2 a \sqrt{\pi}} \cdot \frac{(2 \omega-1)^{\frac{3}{2}} \cdot[\gamma(t)]^{\frac{2 \omega-2}{\omega}} \cdot\left\{[\gamma(\tau)]^{\frac{1}{\omega}}\right\}^{\prime}}{\left(t^{2 \omega-1}-\tau^{2 \omega-1}\right)^{\frac{1}{2}}} \cdot \exp \left\{-\frac{(2 \omega-1)\left([\gamma(t)]^{\frac{2 \omega-1}{\omega}}-[\gamma(\tau)]^{\frac{2 \omega-1}{\omega}}\right)^{2}}{4 a^{2}\left([\gamma(t)]^{\frac{2 \omega-1}{\omega}}-[\gamma(\tau)]^{\frac{2 \omega-1}{\omega}}\right)}\right\} \\
& K_{h}^{(3)}(t, \tau)=-\frac{2}{2 a \sqrt{\pi}} \cdot \frac{(2 \omega-1)^{\frac{3}{2}} \cdot[\gamma(t)]^{\frac{2 \omega-2}{\omega}} \cdot\left\{[\gamma(\tau)]^{\frac{1}{\omega}}\right\}^{\prime}}{\left(t^{2 \omega-1}-\tau^{2 \omega-1}\right)^{\frac{1}{2}}} \cdot \exp \left\{-\frac{(2 \omega-1)\left([\gamma(t)]^{\frac{2 \omega-1}{\omega}}+[\gamma(\tau)]^{\frac{2 \omega-1}{\omega}}\right)^{2}}{4 a^{2}\left([\gamma(t)]^{\frac{2 \omega-1}{\omega}}-[\gamma(\tau)]^{\frac{2 \omega-1}{\omega}}\right)}\right\} \\
& K_{h}^{(4)}(t, \tau)=\frac{2}{a \sqrt{\pi}} \cdot \frac{(2 \omega-1)^{\frac{3}{2}} \cdot[\gamma(t)]^{\frac{2 \omega-2}{\omega}} \cdot\left\{[\gamma(\tau)]^{\frac{1}{\omega}}\right\}^{\prime}}{\left(t^{2 \omega-1}-\tau^{2 \omega-1}\right)^{\frac{1}{2}}} \cdot \exp \left\{-\frac{(2 \omega-1)\left([\gamma(t)]^{\frac{2 \omega-1}{\omega}}-[\gamma(\tau)]^{\frac{2 \omega-1}{\omega}}\right)^{2}}{4 a^{2}\left([\gamma(t)]^{\frac{2 \omega-1}{\omega}}-[\gamma(\tau)]^{\frac{2 \omega-1}{\omega}}\right)}\right\}
\end{aligned}
$$

Next, we show that it is indeed characteristic equation for the equation (7). Firstly, we note that the kernel $K_{h}(t, \tau)$ has the property:

$$
\lim _{t \rightarrow 0} \int_{0}^{t} K_{\gamma}^{(1)}(t, \tau) d \tau=1 .
$$

Equation (8), using the following the change of variables:

$$
\begin{aligned}
& \gamma(t)=\left(\frac{1}{2 \omega-1} \cdot t_{1}\right)^{\frac{\omega}{2 \omega-1}}, \quad \gamma(t)=\left(\frac{1}{2 \omega-1} \cdot \tau_{1}\right)^{\frac{\omega}{2 \omega-1}}, \\
& \varphi\left[\left(\frac{1}{2 \omega-1} \cdot t_{1}\right)^{\frac{\omega}{2 \omega-1}}\right]=\varphi_{1}\left(t_{1}\right), \quad g\left[\left(\frac{1}{2 \omega-1} \cdot t_{1}\right)^{\frac{\omega}{2 \omega-1}}\right]=g_{1}\left(t_{1}\right),
\end{aligned}
$$

reduces to the following integral equation [8]:

$$
\varphi_{1}\left(t_{1}\right)+\int_{0}^{t_{1}} \sqrt{\frac{t_{1}}{\tau_{1}}} K_{1}\left(t_{1}, \tau_{1}\right) \varphi_{1}\left(\tau_{1}\right) d \tau_{1}=g_{1}\left(t_{1}\right) .
$$

The kernel $K_{1}\left(t_{1}, \tau_{1}\right)$ has the form:

$$
K_{1}\left(t_{1}, \tau_{1}\right)=\sum_{i=1}^{4} K_{1}^{(i)}\left(t_{1}, \tau_{1}\right),
$$


where

$$
\begin{aligned}
& K_{1}^{(1)}\left(t_{1}, \tau_{1}\right)=\frac{1}{2 a \sqrt{\pi}} \cdot \frac{t_{1}+\tau_{1}}{\left(t_{1}-\tau_{1}\right)^{\frac{3}{2}}} \cdot \exp \left\{-\frac{\left(t_{1}+\tau_{1}\right)^{2}}{4 a^{2}\left(t_{1}-\tau_{1}\right)}\right\} ; \\
& K_{1}^{(2)}\left(t_{1}, \tau_{1}\right)=-\frac{1}{2 a \sqrt{\pi}} \cdot \frac{t_{1}-\tau_{1}}{\left(t_{1}-\tau_{1}\right)^{\frac{3}{2}}} \cdot \exp \left\{-\frac{\left(t_{1}-\tau_{1}\right)^{2}}{4 a^{2}\left(t_{1}-\tau_{1}\right)}\right\} ; \\
& K_{1}^{(3)}\left(t_{1}, \tau_{1}\right)=-\frac{2}{a \sqrt{\pi}} \cdot \frac{1}{\left(t_{1}-\tau_{1}\right)^{\frac{1}{2}}} \cdot \exp \left\{-\frac{\left(t_{1}+\tau_{1}\right)^{2}}{4 a^{2}\left(t_{1}-\tau_{1}\right)}\right\} ; \\
& K_{1}^{(4)}\left(t_{1}, \tau_{1}\right)=\frac{2}{a \sqrt{\pi}} \cdot \frac{1}{\left(t_{1}-\tau_{1}\right)^{\frac{1}{2}}} \cdot \exp \left\{-\frac{\left(t_{1}-\tau_{1}\right)^{2}}{4 a^{2}\left(t_{1}-\tau_{1}\right)}\right\}
\end{aligned}
$$

Solution of integral equation (9) has the form [8]:

$$
\varphi\left(t_{1}\right)=g\left(t_{1}\right)+\int_{0}^{t} \sqrt{\frac{t_{1}}{\tau_{1}}} \cdot R\left(t_{1}, \tau_{1}\right) \cdot g\left(t_{1}\right) d \tau_{1}+C \cdot \varphi_{\text {hom }}\left(t_{1}\right),
$$

which also belongs to the class $L_{\infty}\left(\mathrm{R}_{+} ; \sqrt{t_{1}} \exp \left\{\frac{t_{1}}{4 a^{2}}\right\}\right)$.

Moreover, the following Lemma holds for the resolvent [8].

Lemma 1. The resolvent $R\left(\mathrm{t}_{1}, \tau_{1}\right)$ admits an estimate

$$
\left|R\left(\mathrm{t}_{1}, \tau_{1}\right)\right| \leq C \frac{\tau_{1}}{\left(t_{1}-\tau_{1}\right)^{3 / 2}} \exp \left\{-\frac{t_{1} \tau_{1}}{a^{2}\left(t_{1}-\tau_{1}\right)}\right\}, \quad 0<\tau_{1}<t_{1}<+\infty .
$$

\section{Solution of characteristic integral equation (8)}

Returning to the old variables, in equality (10), we obtain the solution of characteristic equation (8):

$$
\varphi(t)=g(t)+\int_{0}^{t}\left(\frac{t}{\tau}\right)^{\frac{3}{2 \omega}-1} R_{h}(t, \tau) g(t) d \tau+C \cdot \varphi_{\mathrm{hom}}\left((2 \omega-1)(2 \omega-1)[\gamma(\tau)]^{\frac{2 \omega-1}{\omega}}\right),
$$

and the resolvent $R_{h}(t, \tau)$ satisfies the estimate

$$
R_{h}(t, \tau) \leq C_{1}(\omega) \cdot \frac{[\gamma(t)]^{\frac{5 \omega-3}{\omega}}\left\{[\gamma(\tau)]^{\frac{1}{\omega}}\right\}^{\prime}}{\left([\gamma(t)]^{\frac{2 \omega-1}{\omega}}-[\gamma(\tau)]^{\frac{2 \omega-1}{\omega}}\right)^{\frac{3}{2}}} \cdot \exp \left\{-\frac{(2 \omega-1)[\gamma(t)]^{\frac{2 \omega-1}{\omega}} \cdot[\gamma(\tau)]^{\frac{2 \omega-1}{\omega}}}{a^{2}\left([\gamma(t)]^{\frac{2 \omega-1}{\omega}}-[\gamma(\tau)]^{\frac{2 \omega-1}{\omega}}\right)}\right\} .
$$

Theorem 1. For any right side $g(t) \in L_{\infty}\left(R ;[\gamma(t)]^{\frac{3}{2 \omega}-1}\right)$ integral equation (8) has a general solution $\varphi(t) \in L_{\infty}\left(R ;[\gamma(t)]^{\frac{3}{2 \omega}-1}\right):$

$$
\varphi(t)=g(t)+\int_{0}^{t}\left(\frac{\gamma(t)}{\gamma(\tau)}\right)^{\frac{3}{2 \omega}-1} R_{h}(t, \tau) g(t) d \tau+C \cdot \varphi_{\mathrm{hom}}\left((2 \omega-1) t^{2 \omega-1}\right),
$$

where $\varphi_{\text {hom }}(t)$ is the solution of the homogeneous equation, and for the resolvent $R_{h}(t, \tau)$ we have estimate (11).

Solution of integral equation (7). (Regularization method for solving the characteristic equation)

Using Remark 2, we consider equation (6), which we represent as:

$$
\varphi(t)+\int_{0}^{t} K_{h}(t, \tau) \varphi(\tau) d \tau=\int_{0}^{t}\left[K_{h}(t, \tau)-K_{\gamma}(t, \tau)\right] \varphi(\tau) d \tau+F(t) .
$$


Assuming the right-hand side of equation (12) is temporarily known, we write its solution as:

$$
\begin{gathered}
\varphi(t)=\int_{0}^{t}\left[K_{h}(t, \tau)-K_{\gamma}(t, \tau)\right] \varphi(\tau) d \tau+\int_{0}^{t} R_{h}(t, \tau)\left\{\int_{0}^{\tau}\left[K_{h}\left(\tau, \tau_{1}\right)-K_{\gamma}\left(\tau, \tau_{1}\right)\right] \varphi\left(\tau_{1}\right) d \tau_{1}\right\} d \tau+ \\
+F(t)+\int_{0}^{t} R_{h}(t, \tau) \cdot F(\tau) d \tau+C_{0} \cdot \varphi_{\mathrm{hom}}\left((2 \omega-1) \cdot t^{2 \omega-1}\right) .
\end{gathered}
$$

In the iterated integral, we change the order of integration and change the roles of the variables $\tau$ and $\tau_{1}$, then we obtain

$$
\varphi(t)+\int_{0}^{t} \overline{\tilde{K}}(t, \tau) \cdot \varphi(\tau) d \tau=C_{0} \cdot \varphi_{\mathrm{hom}}\left((2 \omega-1) \cdot t^{2 \omega-1}\right)+\hat{F}(t)
$$

The kernel $\overline{\tilde{K}}(t, \tau)$ has the form:

$$
\overline{\tilde{K}}(t, \tau)=\tilde{K}(t, \tau)+\bar{K}(t, \tau)
$$

where

$$
\tilde{K}(t, \tau)=K_{h}(t, \tau)-K_{\gamma}(t, \tau), \quad \bar{K}(t, \tau)=\int_{\tau_{1}}^{t} R\left(t, \tau_{1}\right)\left[K_{h}\left(\tau_{1}, \tau\right)-K_{\gamma}\left(\tau_{1}, \tau\right)\right] d \tau_{1} .
$$

Let's introduce the following notations:

$$
K_{h}^{(i)}(t, \tau)=P_{h}^{(i)} \exp \left\{-Q_{h}^{(i)}\right\} ; \quad K_{\gamma}^{(i)}(t, \tau)=P_{\gamma}^{(i)} \exp \left\{-Q_{\gamma}^{(i)}\right\}, \quad i=1,2,3,4,
$$

where

$$
\begin{gathered}
P_{h}^{(1)}(t, \tau)=\frac{1}{2 a \sqrt{\pi}} \cdot \frac{(2 \omega-1)^{\frac{3}{2}}\left([\gamma(\tau)]^{\frac{2 \omega-1}{\omega}} \cdot[\gamma(t)]^{\frac{2 \omega-2}{\omega}}+[\gamma(t)]^{\frac{4 \omega-3}{\omega}}\right)\left\{[\gamma(\tau)]^{\frac{1}{\omega}}\right\}^{\prime}}{\left([\gamma(t)]^{\frac{2 \omega-1}{\omega}}-[\gamma(\tau)]^{\frac{2 \omega-1}{\omega}}\right)^{\frac{3}{2}}}, \\
Q_{h}^{(1)}(t, \tau)=\frac{(2 \omega-1)\left([\gamma(t)]^{\frac{2 \omega-1}{\omega}}+[\gamma(\tau)]^{\frac{2 \omega-1}{\omega}}\right)^{2}}{4 a^{2}\left([\gamma(t)]^{\frac{2 \omega-1}{\omega}}-[\gamma(\tau)]^{\frac{2 \omega-1}{\omega}}\right)} \\
P_{\gamma}^{(1)}(t, \tau)=\frac{1}{2 a \sqrt{\pi}} \cdot \frac{\gamma(t)+\gamma(\tau)}{(t-\tau)^{\frac{3}{2}}}, \quad Q_{\gamma}^{(1)}(t, \tau)=\frac{(\gamma(t)+\gamma(\tau))^{2}}{4 a^{2}(t-\tau)}
\end{gathered}
$$

Now we prove the following theorem.

Theorem 2. If function $\gamma(t)=\left[t\left(1+\alpha_{0}(t)\right)\right]^{\omega}$, where $\alpha_{0}(t)=t^{\beta} \sigma(t), \quad \beta>0$, and function $\sigma(t)$ is twice continuously differentiable for $0<t<\infty$, and $|\sigma(t)| \leq C, \sigma(t) \neq 0$, then we have an estimate:

$$
\left|K_{\gamma}(t, \tau)-K_{h}(t, \tau)\right| \leq C(\omega) \frac{t^{\omega-1}}{\sqrt{t-\tau}} \times\left[\exp \left(-Q_{\gamma}(t, \tau) / 2\right)+\exp \left(-Q_{h}(t, \tau) / 2\right)\right]
$$

and the limit relation:

$$
\lim _{t \rightarrow+0} \int_{0}^{t}\left[K_{\gamma}(t, \tau)-K_{h}(t, \tau)\right] d \tau=0
$$

holds.

Note that estimate (14) is obvious for the terms

$$
\left|K_{h}^{(i)}(t, \tau)-K_{\gamma}^{(i)}(t, \tau)\right| \text { for } i=2,3,4 .
$$

Now we prove the estimate (14) for $i=1$. 
Lemma 2. If $\alpha_{0}(t)$ is monotonically increasing function, then the inequalities:

$$
\begin{gathered}
2 \omega-1 \leq \frac{\left[t\left(1+\alpha_{0}(t)\right)\right]^{2 \omega-1}-\left[\tau\left(1+\alpha_{0}(\tau)\right)\right]^{2 \omega-1}}{\left[t\left(1+\alpha_{0}(t)\right)\right]^{2 \omega-2}\left(1+\alpha_{0}\left(t_{1}\right)+t_{1} \alpha_{0}^{\prime}\left(t_{1}\right)\right)(t-\tau)} \leq 1 \text { for } \frac{1}{2}<\omega<1, \\
1 \leq \frac{\left[t\left(1+\alpha_{0}(t)\right)\right]^{2 \omega-1}-\left[\tau\left(1+\alpha_{0}(\tau)\right)\right]^{2 \omega-1}}{\left[t\left(1+\alpha_{0}(t)\right)\right]^{2 \omega-2}\left(1+\alpha_{0}\left(t_{1}\right)+t_{1} \alpha_{0}^{\prime}\left(t_{1}\right)\right)(t-\tau)} \leq 2 \omega-1 \text { for } \omega \geq 1,
\end{gathered}
$$

hold, where $t_{1}=\tau+\theta_{1}(t-\tau), \quad 0<\theta_{1}<1$.

Proof of the Lemma 2. It's obvious that [20;456]:

$$
\begin{gathered}
2 \omega-1 \leq \frac{1-x^{2 \omega-1}}{1-x} \leq 1, \quad \text { if } \quad \frac{1}{2}<\omega<1, \quad 0 \leq x \leq 1 ; \\
1 \leq \frac{1-x^{2 \omega-1}}{1-x} \leq 2 \omega-1, \quad \text { if } \quad \omega \geq 1, \quad 0 \leq x \leq 1 .
\end{gathered}
$$

Let $\omega \geq 1$, then for $0 \leq \tau \leq t$ we get

$$
\begin{gathered}
{\left[t\left(1+\alpha_{0}(t)\right)\right]^{2 \omega-2}\left(t-\tau+t \alpha_{0}(t)-\tau \alpha_{0}(\tau)\right) \leq\left[t\left(1+\alpha_{0}(t)\right)\right]^{2 \omega-1}-\left[\tau\left(1+\alpha_{0}(\tau)\right)\right]^{2 \omega-1} \leq} \\
\leq(2 \omega-1)\left[t\left(1+\alpha_{0}(t)\right)\right]^{2 \omega-2}\left(t-\tau+t \alpha_{0}(t)-\tau \alpha_{0}(\tau)\right) .
\end{gathered}
$$

Using Lagrange's interpolation formula, we have

$$
t \alpha_{0}(t)-\tau \alpha_{0}(\tau)=\left(\alpha_{0}\left(t_{1}\right)+t_{1} \alpha_{0}^{\prime}\left(t_{1}\right)\right)(t-\tau),
$$

where $t_{1}=\tau+\theta_{1}(t-\tau), 0<\theta_{1}<1$.

The proof is analogously for the case $\frac{1}{2}<\omega<1$.

The following lemmas are proved in a similar way.

Lemma 3. If the function $\gamma(t)=\left[t\left(1+\alpha_{0}(t)\right)\right]^{\omega}$, where $\alpha_{0}(t)=t^{\beta} \sigma(t), \beta>0$ and the function $\alpha_{0}(t)$ increases monotonically for $0<t<\infty$, and $|\sigma(t)| \leq C$, then estimate:

$$
\left|P_{h}^{(1)}(t, \tau)-P_{\gamma}^{(1)}(t, \tau)\right| \leq C_{2}(\omega) \frac{t^{\omega+\beta}}{(t-\tau)^{\frac{3}{2}}}
$$

holds.

Lemma 4. Under the conditions of Lemma 3, estimate:

$$
\left|Q_{h}^{1}(t, \tau)-Q_{\gamma}^{1}(t, \tau)\right| \leq M_{1} \frac{t^{2 \omega+\beta}}{t-\tau}+M_{2} t^{2 \omega-1}
$$

holds.

Proof of the Theorem 2. First, we establish the following inequality:

$$
P_{2}(t, \tau)=\cdot \frac{\left[t\left(1+\alpha_{0}(t)\right)\right]^{\omega}}{2 \sqrt{\pi}(t-\tau)^{3 / 2}} \leq M_{3}(\omega) \cdot \frac{t^{\omega}}{(t-\tau)^{3 / 2}} .
$$

For those values of parameter $\omega, 0<\tau<t<\infty$, for which $\left|Q_{h}^{1}(t, \tau)-Q_{\gamma}^{1}(t, \tau)\right|>0$, the required estimate follows from the following inequalities:

$$
\begin{gathered}
\left|K_{h}^{1}(t, \tau)-K_{\gamma}^{1}(t, \tau)\right| \leq\left|\left(P_{h}^{1}(t, \tau)-P_{\gamma}^{1}(t, \tau)\right) \exp \left\{-Q_{\gamma}(t, \tau)\right\}\right|+ \\
+\left|P_{\gamma}^{1}(t, \tau) \exp \left\{-Q_{h}^{1}(t, \tau)\right\}\left(1-\exp \left\{-Q_{\gamma}^{1}(t, \tau)+Q_{h}^{1}(t, \tau)\right\}\right)\right| \leq \\
\leq\left|P_{h}^{1}(t, \tau)-P_{\gamma}^{1}(t, \tau)\right| \exp \left\{-Q_{\gamma}^{1}(t, \tau)\right\}+\left|P_{\gamma}^{1}(t, \tau)\left(Q_{\gamma}^{1}(t, \tau)-Q_{h}^{1}(t, \tau)\right) \exp \left\{-Q_{h}^{1}(t, \tau)\right\}\right| .
\end{gathered}
$$

Hence, taking into account the Lemmas $2-4$, we get:

$$
\left|K_{h}-K_{\gamma}\right| \leq\left\{\bar{M} \frac{t^{\omega+\beta}}{(t-\tau)^{\frac{3}{2}}}+M_{3} \frac{t^{\omega}}{(t-\tau)^{\frac{3}{2}}}\left(M_{1} \frac{t^{2 \omega+\beta}}{t-\tau}+M_{2} t^{2 \omega-1}\right)\right\} e^{-Q_{h}} \leq
$$




$$
\begin{gathered}
\leq \cdot \frac{t^{\omega-1}}{(t-\tau)^{\frac{1}{2}}}\left(\bar{M} \frac{t^{\beta+1}}{t-\tau}+\bar{M}_{1} \frac{t^{2 \omega+\beta+1}}{(t-\tau)^{2}}+\bar{M}_{2} \frac{t^{2 \omega}}{t-\tau}\right) \cdot e^{-Q_{h}} \leq \\
\leq \\
\frac{t^{\omega-1}}{\sqrt{t-\tau}}\left(\frac{t}{t-\tau} \cdot e^{\frac{-Q_{h}}{2}} \cdot \bar{M} t^{\beta}+\frac{t^{2}}{(t-\tau)^{2}} e^{\frac{-Q_{h}}{2}} \cdot \bar{M}_{1} t^{2 \omega+\beta-1}+\right. \\
\left.+\frac{t}{t-\tau} e^{\frac{-Q_{h}}{2}} \cdot \bar{M}_{2} t^{2 \omega-1}\right) e^{\frac{-Q_{h}}{2}} \leq C(\omega)\left[\frac{\alpha(t)}{\alpha(\tau)}\right]^{\frac{3}{2}-\omega} \cdot \frac{t^{\omega-1}}{\sqrt{t-\tau}} e^{\frac{-Q_{h}}{2}} .
\end{gathered}
$$

If the values of the parameter $\omega$ and $0<\tau<t<\infty$ are such that the difference $Q_{\gamma}(t, \tau)-Q_{h}(t, \tau)<0$, then it is enough in the same inequalities to interchange the functions $Q_{\gamma}(t, \tau)$ and $Q_{h}(t, \tau), P_{\gamma}(t, \tau)$ and $P_{h}(t, \tau)$ accordingly.

The validity of inequality (14) shows that the difference $K_{\gamma}(t, \tau)-K_{h}(t, \tau)$ has a weak singularity and the limit relation (15) holds.

$$
\lim _{t \rightarrow+0} \int_{0}^{t} \frac{t^{\omega-1}}{\sqrt{t-\tau}}\left[\exp \left(-Q_{h}(t, \tau) / 2\right)+\exp \left(-Q_{\gamma}(t, \tau) / 2\right)\right] d \tau=0 .
$$

Consequently, equation (8) is indeed characteristic equation for equation (7). Theorem 2 is proved.

In order to obtain estimates for $\bar{K}(t, \tau)$, and at the same time for $\overline{\tilde{K}}(t, \tau)$ estimate for the resolvent, we represent in the form:

$$
\left|R\left\{\frac{1}{2 \omega-1}\left([\alpha(\eta)]^{\frac{1-2 \omega}{\omega}}-[\alpha(t)]^{\frac{1-2 \omega}{\omega}}\right)\right\}\right| \leq \tilde{M}_{2} \frac{t^{3 / 2} \eta^{\frac{3}{2}(2 \omega-1)}}{(t-\eta)^{3 / 2}} \exp \left(-C_{2}(\omega) \frac{t \eta^{2 \omega-1}}{t-\eta}\right) .
$$

Here $C_{j}(\omega), \tilde{M}_{j}(\omega), j=1,2$ are constants depending only on $\omega$.

Using estimates (14) and (16), we obtain the following theorem:

Theorem 3. If function $\gamma(t)=\left[t\left(1+\alpha_{0}(t)\right)\right]^{\omega}$, where $\alpha_{0}(t)=t^{\beta} \sigma(t), \beta>0$, and the function $\sigma(t)$ is twice continuously differentiable for $0<t<\infty$, and $|\sigma(t)| \leq C, \sigma(t) \neq 0$, then the kernel $\tilde{K}(t, \tau)$ has a weak singularity, i.e. we have an estimate:

$$
|\overline{\tilde{K}}(t, \tau)| \leq \frac{t^{1 / 2+\varepsilon}}{\tau^{3 / 2-\omega+\varepsilon}(t-\tau)^{1 / 2}}, 0<\varepsilon<\omega-\frac{1}{2}, 0<\tau<t<\infty,
$$

which means that integral equation (7) for any $f(t),[\gamma(t)]^{\frac{3}{2 \omega-1}} \cdot f(t) \in L_{\infty}\left(R_{+}\right)$has a unique nonzero solution:

$$
\varphi(t) \in L_{\infty}\left(R_{\infty} ;[\gamma(t)]^{3 / 2 \omega-1}\right)
$$

Proof. Since $\overline{\tilde{K}}(t, \tau)=\tilde{K}(t, \tau)+\bar{K}(t, \tau)$, then estimate (17) follows from (14), estimates for resolvent (16) and below relations. Using the following double inequality $[19 ; 55]$ :

$$
C_{1} t^{\rho-1}(t-\tau) \leq t^{\rho}-\tau^{\rho} \leq C_{2} \tau^{\rho-1}(t-\tau) \text {, где } C_{1}=\min \{1, \rho\}, C_{2}=\max \{1, \rho\},
$$

first we get $(\rho=2 \omega-1)$ :

$$
\bar{K}(t, \tau) \leq M_{2}(\omega) \int_{\tau}^{t} \eta^{-\gamma-1}\left(\frac{\eta}{\tau}\right)^{1-\gamma / 2} \frac{\eta^{\omega-1}}{\sqrt{\eta-\tau}} \frac{t^{3 / 2} \eta^{3 \omega 3 / 2}}{(t-\eta)^{3 / 2}} \exp \left(-C_{2}(\omega) \frac{t \eta^{2 \omega-1}}{t-\eta}\right) d \eta=I_{2}(t, \tau) .
$$

We represent the function $I_{2}$ as a sum of two terms:

$$
I_{2}(t, \tau)=I_{21}(t, \tau)+I_{22}(t, \tau)
$$

for each of which we will have:

$$
\leq \frac{1}{\sqrt{t-\tau}}\left[C_{1}(\omega)+C_{2}(\omega)(\tau / t)^{\varepsilon} \cdot \ln |\tau / t| \cdot(t / \tau)^{\varepsilon}\right]=\frac{1}{\sqrt{t-\tau}}\left[C_{1}(\omega)+C_{3}(\omega)(t / \tau)^{\varepsilon}\right]
$$


For the second term

$$
\begin{gathered}
I_{22}(t, \tau)=\int_{\frac{t+\tau}{2}}^{t} \frac{t \eta^{\omega-1}}{\sqrt{\eta-\tau}(t-\eta)^{\frac{3}{2}}} \exp \left(-C_{2}(\omega) \frac{t \eta^{2 \omega-1}}{t-\eta}\right) d \eta \leq \\
\leq \frac{C(\omega)}{\sqrt{t-\tau}} \int_{\frac{t+\tau}{2}}^{t} \frac{t \eta^{\omega-1}}{(t-\eta)^{\frac{3}{2}}} \exp \left(-C_{3}(\omega) \frac{t(t+\tau)^{2 \omega-1}}{t-\eta}\right) d \eta \leq \\
\leq \frac{C(\omega)}{\sqrt{t-\tau}} \int_{\frac{t+\tau}{2}}^{t} \frac{t \eta^{\omega-1}}{(t-\eta)^{\frac{3}{2}}} \exp \left(-C_{3}(\omega) \frac{t^{2 \omega}}{t-\eta}\left\{1+\frac{\tau}{t}\right\}^{2 \omega-1}\right) d \eta \leq \\
\leq \frac{C(\omega)}{\sqrt{t-\tau}} \int_{0}^{t} \frac{t^{\omega}}{(t-\eta)^{\frac{3}{2}}} \exp \left(-C_{4}(\omega) \frac{t^{2 \omega}}{t-\eta}\right) d \eta=\frac{C_{5}(\omega)}{\sqrt{t-\tau}} \int_{t^{\frac{2 \omega-1}{2}}}^{\infty} \exp \left\{-z^{2}\right\} d z \leq \frac{C_{5}(\omega)}{\sqrt{t-\tau}} .
\end{gathered}
$$

In these inequalities, the constants $C(\omega), C_{j}(\omega), j=1,2,3,4,5$ are different and depend only on $\omega$. The obtained inequalities imply the required estimate (17). This completes the proof of the Theorem.

Remark 3. From relation (13) it follows that homogeneous equation

$$
\varphi(t)-\int_{0}^{t} K_{\omega}(t, \tau) \mu(\tau) d \tau=0, t \in R_{+},
$$

is equivalent to the nonhomogeneous equation:

$$
\varphi(t)+\int_{0}^{t} \overline{\tilde{K}}(t, \tau) \cdot \varphi(\tau) d \tau=C_{0} \cdot \varphi_{\mathrm{hom}}\left((2 \omega-1) \cdot t^{2 \omega-1}\right) .
$$

Study of the boundary value problem

A solution of the original boundary value problem (1)-(2) have the form:

$$
u(x, t)=\int_{0}^{x} v(\xi, t) d \xi
$$

where $v(x, t)=v_{\text {hom }}(x, t)+v_{\text {part }}(x, t)$, and

$$
\begin{gathered}
v_{\text {hom }}(x, t)=\frac{1}{2 a \sqrt{\pi}} \int_{0}^{t} \frac{1}{(t-\tau)^{1 / 2}}\left[-\exp \left\{-\frac{(x+\gamma(\tau))^{2}}{4 a^{2}(t-\tau)}\right\}+\exp \left\{-\frac{(x-\gamma(\tau))^{2}}{4 a^{2}(t-\tau)}\right\}\right] \cdot \varphi_{0}(\tau) d \tau \\
v_{\text {part }}(x, t)=\frac{1}{2 a \sqrt{\pi}} \int_{0}^{t} \frac{1}{(t-\tau)^{1 / 2}}\left[-\exp \left\{-\frac{(x+\gamma(\tau))^{2}}{4 a^{2}(t-\tau)}\right\}+\exp \left\{-\frac{(x-\gamma(\tau))^{2}}{4 a^{2}(t-\tau)}\right\}\right] \cdot \varphi_{\text {part }}(\tau) d \tau+ \\
+\frac{1}{2 a \sqrt{\pi}} \int_{0}^{t} \int_{0}^{\infty} \frac{1}{(t-\tau)^{1 / 2}}\left[-\exp \left\{-\frac{(x+\xi)^{2}}{4 a^{2}(t-\tau)}\right\}+\exp \left\{-\frac{(x-\xi)^{2}}{4 a^{2}(t-\tau)}\right\}\right] \cdot \tilde{f}(\xi, \tau) d \xi d \tau+ \\
\quad+\frac{1}{2 a \sqrt{\pi}} \int_{0}^{t} \frac{x}{(t-\tau)^{3 / 2}} \exp \left\{-\frac{x^{2}}{4 a^{2}(t-\tau)}\right\} v_{0}(\tau) d \tau
\end{gathered}
$$

where the functions $(\gamma(t))^{\frac{3 / 2-\omega}{\omega}} \cdot \varphi(t)$ and $(\gamma(t))^{\frac{3 / 2-\omega}{\omega}} \cdot \tilde{f}(x, t)$ are bounded and continuous functions on $R_{+}$and $Q$, respectively.

From (18)-(20) we obtain the following estimates:

$$
u_{h o m}(x, t) \leq a \sqrt{\pi}\left\{-2 \operatorname{erf}\left(\frac{x}{2 a \sqrt{2 \omega-1} \cdot[\gamma(t)]^{\frac{2 \omega-1}{2 \omega}}}\right)+\right.
$$

Mathematics series. № 1(101)/2021 


$$
\begin{gathered}
+2 \exp \left\{\frac{(2 \omega-1)[\gamma(t)]^{\frac{2 \omega-1}{\omega}}}{4 a^{2}}\right\} \operatorname{erfc}\left(\frac{\sqrt{2 \omega-1} \cdot[\gamma(t)]^{\frac{2 \omega-1}{2 \omega}}}{2 a}\right)- \\
-\exp \left\{\frac{2 x+(2 \omega-1)[\gamma(t)]^{\frac{2 \omega-1}{\omega}}}{4 a^{2}}\right\} \operatorname{erfc}\left(\frac{x+(2 \omega-1)[\gamma(t)]^{\frac{2 \omega-1}{\omega}}}{2 a \sqrt{2 \omega-1} \cdot[\gamma(t)]^{\frac{2 \omega-1}{2 \omega}}}\right)- \\
\left.-\exp \left\{-\frac{2 x-(2 \omega-1)[\gamma(t)]^{\frac{2 \omega-1}{\omega}}}{4 a^{2}}\right\} \operatorname{erfc}\left(-\frac{x-(2 \omega-1)[\gamma(t)]^{\frac{2 \omega-1}{\omega}}}{2 a \sqrt{2 \omega-1}[\gamma(t)]^{\frac{2 \omega-1}{2 \omega}}}\right)\right\} .
\end{gathered}
$$

For $u_{\text {part }}(x, t)$, we have that

$$
\begin{gathered}
u_{\text {part }}(x, t) \leq a \sqrt{\pi}\left\{-2 \operatorname{erf}\left(\frac{x}{2 a \sqrt{2 \omega-1 \cdot} \cdot[\gamma(t)]^{\frac{2 \omega-1}{2 \omega}}}\right)+\right. \\
+2 \exp \left\{\frac{(2 \omega-1)[\gamma(t)]^{\frac{2 \omega-1}{\omega}}}{4 a^{2}}\right\} \operatorname{erfc}\left(\frac{\sqrt{2 \omega-1} \cdot[\gamma(t)]^{\frac{2 \omega-1}{2 \omega}}}{2 a}\right)- \\
-\exp \left\{\frac{2 x+(2 \omega-1)[\gamma(t)]^{\frac{2 \omega-1}{\omega}}}{4 a^{2}}\right\} \operatorname{erfc}\left(\frac{x+(2 \omega-1)[\gamma(t)]^{\frac{2 \omega-1}{\omega}}}{2 a \sqrt{2 \omega-1} \cdot[\gamma(t)]^{\frac{2 \omega-1}{2 \omega}}}\right)- \\
\left.-\exp \left\{-\frac{2 x-(2 \omega-1)[\gamma(t)]^{\frac{2 \omega-1}{\omega}}}{4 a^{2}}\right\} \operatorname{erfc}\left(-\frac{x-(2 \omega-1)[\gamma(t)]^{\frac{2 \omega-1}{\omega}}}{2 a \sqrt{2 \omega-1}[\gamma(t)]^{\frac{2 \omega-1}{2 \omega}}}\right)\right\}+\frac{1}{\sqrt{\pi}} \operatorname{erf}\left(\frac{\sqrt{2 \omega-1}[\gamma(t)]^{\frac{2 \omega-1}{2 \omega}}}{2 a}\right) .
\end{gathered}
$$

Therefore estimates of these integrals give the statement of the Theorem.

Theorem 5. For any right side $f(t) \in L_{\infty}\left(\mathrm{R}_{+} ;[\gamma(t)]^{\frac{3 / 2-\omega}{\omega}} \exp \left\{\gamma(t) /\left(4 a^{2}\right)\right\}\right)$ and for given functions $f(x, t) \in W_{\infty}^{1,0}\left(G ;[\gamma(t)]^{3 / 2 \omega-1} \exp \left\{[\gamma(t)]^{\frac{2 \omega-1}{\omega}} /\left(4 a^{2}\right)\right\}\right), u_{0}(t) \in L_{\infty}\left(R_{+} ;[\gamma(t)]^{\frac{\omega-3 / 2}{\omega}}\right) ; u_{1}(t) \in L_{\infty}\left(\mathrm{R}_{+}\right.$; $[\gamma(t)]^{\frac{3 / 2-\omega}{\omega}}$ ) boundary value problem $(1)-(2)$ has a general solution $u(x, t) \in L_{\infty}\left(G ;\left(x+[\gamma(t)]^{3 / 2 \omega-1}\right)^{-1}\right)$, which is determined from formula (18)-(20).

\section{Acknowledgments}

Supported by the grant projects AP08956033 (2020 - 2021) and AP08855372 (2020 - 2022) from the Ministry of Science and Education of the Republic of Kazakhstan.

\section{References}

1 Карташов Э.М. Аналитические методы в теории теплопроводности твердых тел / Э.М. Карташов. - М.: Высш. шк., 1985. - 553 с.

2 Карташов Э.М. Аналитические методы решения краевых задач уравнения теплопроводности в области с движущимися границами / Э.М. Карташов, Б.Я. Любов // Изв. АН СССР. Энергетика и транспорт. - 1974. - № 6. - С. 83-111.

3 Карташов Э.М. Аналитические методы решения краевых задач нестационарной теплопроводности в областях с движущимися границами / Э.М. Карташов // Изв. РАН. Энергетика. $-1999 .-$ № 5. - C. 3-34.

4 Карташов Э.М. Аналитические методы решения краевых задач нестационарной теплопроводности в областях с движущимися границами / Э.М. Карташов // Инж.-физ. журн. $-2001 .-74 .-$ № 2. - C. 171-195. DOI: 10.1023/A:1016641613982

5 Аттетков А.В. Температурное поле полупространства с термически тонким покрытием в импульсных режимах теплообмена с окружающей средой / А.В. Аттетков, Р.А. Власов, И.К. Волков // Инж.-физ. журн. - 2001. - 74. - № 3. - C. 647-655. DOI: 10.1023/A:1016756227188

6 Kharin S.N. Mathematical models of phenomena in electrical contacts: Monograph / S.N. Kharin // A.P. Ershov Institute of Informatics system, Siberian Branch of RAS. - 2017. - 193. 
7 Amangaliyeva M.M. On one homogeneous problem for the heat equation in an infinite angular domain / M.M. Amangaliyeva, M.T. Jenaliyev, M.T. Kosmakova, M.I. Ramazanov // Sib. Math. Jour. - 2015. 56. - № 6. - P. 82-995.

8 Jenaliev M.T. On a homogeneous parabolic problem in an infinite angular domain / M.T. Jenaliev, S.A. Iskakov, M.I. Ramazanov // Eurasian journal of mathematical and computer applications. - 2019. - 7. - № 1. - P. 38-52.

9 Шишкин А.Г. Математическое моделирование физических процессов в термоядерной и газоразрядной плазме / А.Г. Шишкин. - М.: Аргамак-медиа, 2015. - 235 с.

10 Намитоков K.K. Математическое моделирование процессов в газоразрядной плазме / K.K. Намитоков, П.Л. Пахомов, С.Н. Харин. - Алма-Ата: Наука, 1988. - 208 с.

11 Карташов Э.М. Проблема теплового удара в области с движущейся границей на основе новых интегральных соотношений / Э.М. Карташов // Изв. РАН. Энергетика. - 1997. - 4. - С. 122-137.

12 Веригин Н.Н. Об одном классе гидромеханических задач для областей с подвижными границами / Н.Н. Веригин // Динамика жидкости со свободными границами. - 1980. - 46. - С. 23-32.

13 Багдасаров Х.С. Тепло- и массоперенос при выращивании монокристаллов направленной кристаллизацией / Х.С. Багдасаров, Л.А. Горяинов. - М.: Физматлит, 2007. - 234 с.

14 Солонников В.А. Одномерная параболическая задача, возникающая при исследовании некоторых задач со свободными границами / В.А. Солонников, А. Фазано // Зап. науч. семин. ПОМИ. - 2000. - 269. - С. 322-338.

15 Тихонов А.Н. Уравнения математической физики: учеб. пос. для ун-тов / А.Н. Тихонов, А.А. Самарский. - 4-е изд., испр. - М.: Наука, 1972. - 735 с.

16 Полянин А.Д. Справочник по интегральным уравнениям / А.Д. Полянин, А.В. Манжиров. - М.: Физматлит, 2003. - 608 с.

17 Jenaliyev M.T. On a Volterra equation of the second kind with 'incompressible' kernel / M.T. Jenaliyev, M.M. Amangaliyeva, M.T. Kosmakova and M.I. Ramazanov // Advances in Difference Equations. 2015. - 71. - P. 14

18 Amangaliyeva M.M. About Dirichlet boundary value problem for the heat equation in the infinite angular domain / M.M. Amangaliyeva, M.T. Jenaliyev, M.T. Kosmakova and M.I. Ramazanov // Boundary Value Problem-. - 2014. - 213. - P. 21.

19 Hardy G.G. Inequalities / G.G. Hardy, J.E. Littlewood, G.M. Polya. - Foreign literature, 1948. - 456 p.

\author{
М. Т. Дженалиев, М.И. Рамазанов, А.О. Танин
}

\title{
Шекарасы $x=\gamma(t)$ заңдылығымен қозғалатын Солонников-Фазан есебінің шешімі туралы
}

\begin{abstract}
Жұмыста бастапқы мезетте жойылатын облыстағы жылуөткізгіштік теңдеу үшін шекаралық есептің шешімі зерттелген. Мұнда, шекарасы уақытқа байланысты $x=\gamma(t)$ заңдылығымен өзгереді. Қарастырылып отырған есеп жалпыланған жылу потенциалдарының көмегімен псевдо-вольтерралық интегралдық теңдеуге келтіріледі. Ал интегралдық оператордың нормасының бірге тең болуы оның ерекшелігі болып табылады. Сонымен қатар, сәйкес біртекті интегралдық теңдеудің нөлдік емес шешімінің болатыны көрсетілген.
\end{abstract}

Кілт сөздер: жылуөткізгіштік теңдеу, жылжымалы шекара, жойылатын облыс, псевдо-вольтерралық интегралдық теңдеу. 
М.Т. Дженалиев, М.И. Рамазанов, А.О. Танин

\title{
К решению задачи Солонникова-Фазано при движении границы по произвольному закону $x=\gamma(t)$
}

\begin{abstract}
В работе исследованы вопросы разрешимости граничной задачи для уравнения теплопроводности в области, которая вырождается в точку в начальный момент времени. При этом изменяющаяся со временем граница движется по произвольному закону $x=\gamma(t)$. С помощью обобщенных тепловых потенциалов исследуемая задача редуцируется к псевдо-вольтерровому интегральному уравнению, особенность которого заключается в том, что норма интегрального оператора равна единице. Показано, что соответствующее однородное интегральное уравнение имеет ненулевое решение.
\end{abstract}

Ключевые слова: уравнение теплопроводности, подвижная граница, вырождающаяся область, псевдовольтерровое интегральное уравнение.

\section{References}

1 Kartashov, E.M. (1985). Analiticheskie metody v teorii teploprovodnosti tverdykh tel [Analytic methods in the theory of thermal conductivity of solids/. Moscow: Vysshaia shkola [in Russian].

2 Kartashov, E.M., \& Liubov B.Ya. (1974). Analiticheskie metody resheniia kraevykh zadach uravneniia teploprovodnosti v oblasti s dvizhushchimisia hranitsami [Analytic methods for solving boundary value problems for the heat equation in a region with moving boundaries]. Izvestiia AN SSSR. Enerhetika $i$ transport - Proceeding of the USSR AS. Power engineering and transport, 6, 83-111 [in Russian].

3 Kartashov, E.M. (1999). Analiticheskie metody resheniia kraevykh zadach nestatsionarnoi teploprovodnosti v oblastiakh s dvizhushchimisia hranitsami [Analytical Methods of Solution of Boundary-Value Problems of Nonstationary Heat Conduction in Region with Moving Boundaries]. Izvestiia RAN. Enerhetika-Proceedings of the RAS. Power engineering, 5, 3-34 [in Russian].

4 Kartashov, E.M. (2001). Analytical methods of solution of boundary-value problems of nonstationary heat conduction in region with moving boundaries. Journal of Engineering Physics and Thermophysics, 74(2), 498-536. DOI: 10.1023/A:1016641613982.

5 Attetkov, A.V., Vlasov, P.A., \& Volkov, I.K. (2001). Temperature field of a half-space with a thermally thin coating in pulse modes of heat exchange with the environment. Journal of Engineering Physics and Thermophysics, 74(3), 647-655. DOI: 10.1023/A:1016756227188.

6 Kharin, S.N. (2017). Mathematical models of phenomena in electrical contacts: Monograph. A.P. Ershov Institute of Informatics system, Siberian Branch of RAS, 193.

7 Amangaliyeva, M.M., Jenaliyev, M.T., Kosmakova, M.T., \& Ramazanov, M.I. (2015). On one homogeneous problem for the heat equation in an infinite angular domain. Sib. Math. Jour., 56(6), 982-995.

8 Jenaliev, M.T., Iskakov, S.A., \& Ramazanov, M.I. (2019). On a homogeneous parabolic problem in an infinite angular domain. Eurasian journal of mathematical and computer applications, 7(1), 38-52.

9 Shishkin, A.G. (2015). Matematicheskoe modelirovanie fizicheskikh protsessov v termoiadernoi i hazorazriadnoi plazme [Mathematical modeling of physical processes in a fuel and gas-fueled process]. Moscow: Arhamak-media [in Russian].

10 Namitokov, K.K., Pakhomov, P.L., \& Kharin, S.N. (1988). Matematicheskoe modelirovanie protsessov v hazorazriadnoi plazme [Mathematical Modeling of Phenomena in Gaseous Plasma Discharge]. Alma-Ata: Nauka [in Russian].

11 Kartashov, E.M. (1997). Problema teplovoho udara v oblasti s dvizhushcheisia hranitsei na osnove novykh intehralnykh sootnoshenii [Heatstroke problem in a region with a moving boundary based on new integral relations]. Izvestiia RAN. Enerhetika - Proceedings of the RAS. Power engineering, 4, 122-137 [in Russian].

12 Verigin, N.N. (1980). Ob odnom klasse hidromekhanicheskikh zadach dlia ispolzovaniia s podvizhnymi hranitsami [On a class of hydromechanical problems for domains with moving boundaries]. Dinamika sploshnoi sredy - Fluid dynamics with free boundaries, 46, 23-32 [in Russian]. 
13 Bagdasarov, Kh.S., \& Goryainov, L.A. (2007). Teplo- i massoperenos pri vyrashchivanii monokristallov napravlennoi kristallizatsiei [Heat and mass transfer during the growth of single crystals by deposited crystallization]. Moscow: FML [in Russian].

14 Solonnikov, V.A., \& Fasano, A. (2000). Odnomernaia parabolicheskaia zadacha, voznikaiushchaia pri issledovanii nekotorykh zadach so svobodnymi hranitsami [One-dimensional parabolic problem arising in the studyof some free boundary problems]. Zapiski nauchnykh seminarov POMI - POMI Scientific Workshop Notes 269, 322-338 [in Russian].

15 Tikhonov, A.N., \& Samarskii, A.A. (1972). Uravneniia matematicheskoi fiziki [Equations of mathematical physics]. Moscow: Nauka [in Russian].

16 Polyanin, A.D., \& Manzhirov, A.V. (2003). Spravochnik po intehralnym uravneniiam /Handbook of integral equations]. Moscow: FML [in Russian].

17 Jenaliyev, M.T., Amangaliyeva, M.M., Kosmakova, M.T., \& Ramazanov, M.I. (2015). On a Volterra equation of the second kind with "incompressible"kernel. Advances in DiferenceEquations, 71, 14.

18 Amangaliyeva, M.M., Jenaliyev, M.T., Kosmakova, M.T. \& Ramazanov, M.I. (2014). About Dirichlet boundary value problem for the heat equation in the innite angular domain. Boundary Value Problem, 213, 21.

19 Hardy, G.G, Littlewood, J.E., \& Polya, G. (1948). Inequalities. Moscow: Foreign literature. 